\title{
Twenty-one-year follow-up of variable onset MELAS syndrome with heteroplasmic nt3243A>G mtDNA mutation: A case report
}

\author{
Wung Joo Song ${ }^{1,2}$, Yoon Jin Lee ${ }^{3}$, Joon Won Kang ${ }^{3}$, Mea Young Chang ${ }^{3}$, Kyu Sang Song ${ }^{4}$, Dae Young Kang ${ }^{4}$, and Sook Za Kim, ${ }^{1,2 * *}$ \\ ${ }^{1}$ KSZ Children's Hospital, Cheongju, Korea \\ ${ }^{2}$ Korea Genetics Research Center, Cheongju, Korea \\ ${ }^{3}$ Department of Pediatrics, Chungnam National University School of Medicine, Daejeon, Korea \\ ${ }^{4}$ Department of Pathology, Chungnam National University School of Medicine, Daejeon, Korea
}

Mitochondrial encephalomyopathy, lactic acidosis, and stroke-like episodes (MELAS) syndrome is a maternally inherited mitochondrial disorder of which m.3243A $>\mathrm{G}$ is the most commonly associated mutation, resulting in an inability to meet the energy requirements of various organs. MELAS poses a diagnostic challenge owing to its multiple organ involvement and great clinical variability due to its heteroplasmic nature. We report three cases from a family who were initially misdiagnosed with myasthenia gravis or undiagnosed. Although there is no optimal consensus treatment approach for patients with MELAS because of the disease's heterogeneity, our 21-year-long therapy regimen of L-arginine, L-carnitine, and coenzyme Q10 supplementation combined with dietary management appeared to provide noticeable protection from the symptoms and complications. Prompt early diagnosis is important, as optimal multidisciplinary management and early intervention may improve outcomes.

Key words: MELAS syndrome, Myasthenia gravis, Lactic acidosis.

\section{Introduction}

Mitochondrial encephalomyopathy, lactic acidosis, and stroke-like episodes (MELAS) is an inherited mitochondrial multisystem disorder with childhood onset, where symptoms frequently appear between the ages of 2 and 10 years [1]. The clinical diagnostic criteria of MELAS are stroke-like episodes prior to age 40 years, seizures, dementia, encephalopathy, lactic acidosis, and ragged red fibers (RRFs) in muscle biopsy specimens [2]. However, the criteria do not encompass the broad spectrum of presentations that are associated with MELAS, especially those with a low mutant ratio and late onset $[1,3,4]$. Therefore, clinical presentations suggestive of MELAS warrant molecular testing $[1,5]$. If molecular studies do not reveal any causal pathogenic variants, if molecular studies do not reveal any causal pathogenic variants, the test results may be false-negative.

In patients with a history of stroke-like episodes, characteristic lesions may be visible on brain imaging, and may help differentiate MELAS from other causes of cerebral ischemia. If clinical suspicion remains, additional testing on other tissues, such as skin fibroblasts or skeletal muscle biopsy specimens, can be performed [5].

Received: 22 October 2018, Revised: 26 January 2019, Accepted: 30 January 2019, Published: 30 June 2019

${ }^{*}$ Corresponding author: Sook Za Kim, M.D., Ph.D. (iD https://orcid.org/0000-0003-0163-4286

Korea Genetics Research Center/KSZ Children's Hospital, 745 Jikji-daero, Heungdeok-gu, Cheongju 28470, Korea.

Tel: +82-43-216-8280, Fax: +82-43-215-8288, E-mail: kimgenee@naver.com

Conflict of interest: The authors declare that they do not have any conflicts of interest.

(ac) This is an open-access article distributed under the terms of the Creative Commons Attribution Non-Commercial License (http://creativecommons.org/licenses/by-nc/4.0/) which permits unrestricted non-commercial use, distribution, and reproduction in any medium, provided the original work is properly cited.

(c) Copyright 2019 by the Korean Society of Medical Genetics and Genomics 
Over 30 pathogenic variants in mitochondrial deoxyribonucleic acid (mtDNA) have been identified to cause MELAS $[3,5,6]$. The most common variant, which accounts for over $80 \%$ of MELAS cases [4], is an mtDNA transfer ribonucleic acid (tRNA) ${ }^{\text {Leu(UUR) }}$ mutation, 3243A $>$ G. This mutation produces a defective mtRNA for leucine, resulting in the impairment of protein synthesis and respiratory chain function.

MELAS severely affects organ systems with high energy demands, including the brain and skeletal muscle [2]. Heteroplasmic mtDNA mutations lead to threshold effects and diverse clinical variability of the disease. This syndrome normally progresses over years, with episodic deterioration marked by strokelike events.

Herein, we describe a 21-year-long follow-up of three cases from a family with the mt.3243A>G mutation. We also review the clinical characteristics, current recommendations for management, and long-term efficacy of L-arginine supplementation.

\section{Case}

\section{Materials and methods}

The patients were two children and their mother from one family with a history of external ophthalmoplegia. Family pedigree analysis (Fig. 1), clinical evaluation, and radiologic testing, including magnetic resonance imaging (MRI), were conducted. Ophthalmologic, audiologic, electrophysiologic, biochemical, molecular, and pulmonary function tests as well as muscle biopsies were also performed.

For light microscopy study, hematoxylin and eosin (HEtE) staining was performed to view fascicular structures and muscle fibers, and Gomori trichrome (GT) staining was used to show connective tissue accumulation, mitochondrial accumulation, and RRFs. Electron microscopy evaluation was employed to find structural alterations and inclusions in the mitochondria.
Sequencing of the patients' protein-coding genes was performed by cycle sequencing according to the manufacturer's instructions. The amount of mutated DNA was detected by restriction fragment length polymorphism analysis using the restriction endonuclease BstNI. Quantitative evaluation of the heteroplasmic mtDNA was carried out on DNA bands that were excised from agarose gels and processed for subsequent scintillation counting.

Plasma and urine amino acid analyses were carried out by ionexchange chromatography. The urine organic acid analysis was performed using gas chromatography-mass spectrometry.

\section{Case history}

\section{1) Case 1}

A 17-year-old girl was brought to our attention by her parents who wanted a second opinion on their daughter's external ophthalmoplegia, weight loss, and fatigue as well as treatment for suspected myasthenia gravis that appeared to be ineffective. The patient was born at full term, with a birth weight of $2.6 \mathrm{~kg}$ and no concerns for her development and growth. From the age of 9 years, her eyelids became droopy, especially in the afternoon, with diurnal variation, where her symptoms would become worse early in the morning and late at night. By the age of 10 years, she had developed diplopia and ptosis, which had gotten progressively worse.

During physical exercise, her muscle weakness worsened; she would experience dizziness, diplopia, ptosis, and general weakness. Subsequent rest improved her symptoms. The acetylcholine receptor antibody test was not readily available at the time, which contributed to her misdiagnosis. A mass in her thymus was suspected to be a thymoma because of a positive Jolly test result and the frequent association of thymomas with myasthenia gravis. According to her medical record, the Tensilon test was not performed. However, after surgical removal of the

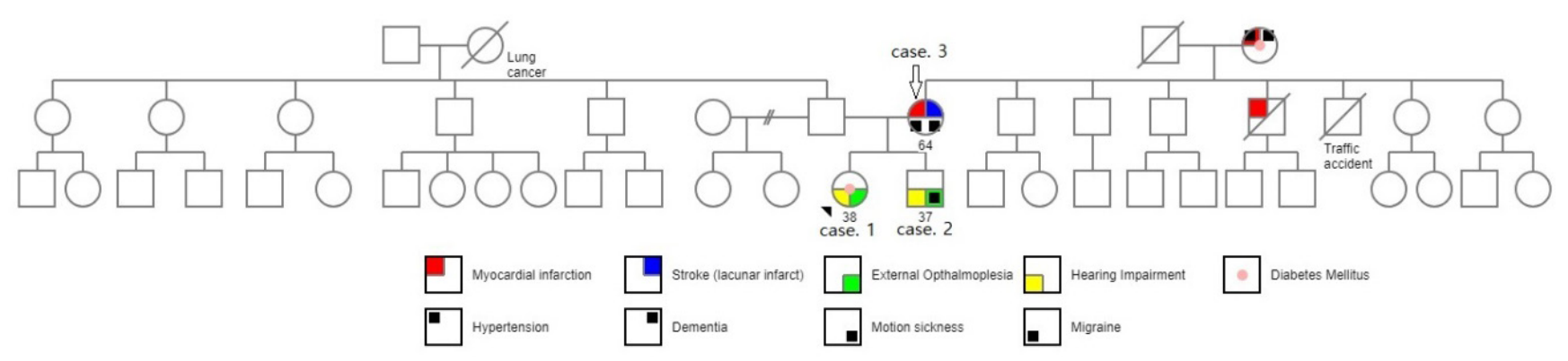

Fig. 1. Pedigree of the family with mitochondrial encephalomyopathy, lactic acidosis, and stroke-like episodes (MELAS) showing typical maternal inheritance. There was no consanguinity. 
tumor mass, the pathologic finding revealed it to be a teratoma $(1.8 \times 1.2 \mathrm{~cm})$, which is not associated with myasthenia gravis.

Her ptosis did not improve at all with the cholinesterase inhibitor treatment, and the drug was eventually discontinued because of severe side effects, including nausea, diarrhea, abdominal pain, and irritability. Her menarche started at the age of 15 years, with an irregular cycle and scant menstruation. Sparse pubic hair and axillary hair were observed 2 years later. At the age of 17 years, she started suffering from tinnitus and sensory neural hearing impairment.

Although she managed to graduate from college, her extreme fatigability was aggravated and she required naps at least twice a day. She continued to experience diurnal variations in eye muscle weakness and fatigability, especially early in the morning and late at night, owing to inadequate calorie intake and long fasting durations that resulted in glycogen depletion. She frequently received intravenous glucose for quick recovery. She has since been on coenzyme 010, vitamin B complex, L-carnitine, and L-arginine supplementation.

At 37 years of age, she developed diabetes mellitus, which was subsequently controlled by oral hypoglycemic agents. She is currently cachectic, with a weight of $27 \mathrm{~kg}$ and height of 149 cm (father's height: $175 \mathrm{~cm}$; mother's height: $153 \mathrm{~cm}$ ). At last follow-up, she appeared tired and her physical examination revealed little subcutaneous fat and prominent ribs. She had droopy eyes, showing limited external ocular muscle control.

Her ophthalmologic examination revealed normal visual acuity, normal fundi without optic atrophy, and limited external ocular muscle movement, especially of the up-gaze and lateral gaze. Neurologic examination showed her to have an alert mental state, good orientation, no dysarthria, and normal cranial nerves. Her sensory and motor functions were normal, as were her deep tendon reflexes.

\section{2) Case 2}

This patient came to our attention when he had nearly identical symptoms as his sister's (Case 1), although his were relatively milder. He complained of stomachache, severe motion sickness during car travel, and fatigue. Like Case 1, he was grossly underweight (weight: $42 \mathrm{~kg}$ ) and short in stature (height: $151 \mathrm{~cm}$ ). His neurologic examination revealed an alert mental state, good memory (including recent and remote memory), good orientation to time and place, and no dysarthria. The muscle strength of his extremities was normal. His craniobulbar strength, including face, eyelids, eye movements, tongue movements, speech, and swallowing, were all normal. He had isocoric pupils, no nystag- mus, and normal fundus. In spite of a mild degree of ptosis, there was an absence of extraocular muscle movement limitation. There was no uvula or tongue deviation with normal gag reflex. His deep tendon reflexes were normal, and the Barbinski test revealed a bilateral plantar response.

He became a physician, is married, and has an 8-month-old healthy son. He elected to have a muscle biopsy done to apply for military duty exemption. The skeletal muscle biopsy showed positive RRFs and a large increase in the number of abnormal mitochondria, many of which had abnormal morphology (crystalline/paracrystalline inclusions of parking lot shape; enlarged, elongated, concentric, thread-like bundles with thickened cristae). At 37 years of age, he complained of severe fatigue, nausea, and recurrent abdominal pain. Progressive fatigue, ptosis, and exercise intolerance were increased.

\section{3) Case 3}

Case 3 is the 64-year-old mother of cases 1 and 2. She was ascertained when her daughter and son were confirmed to have MELAS syndrome. She suffered from frequent migraines, severe nasal allergy, insomnia, poor appetite, and easy fatigability. Her body weight and height were $40 \mathrm{~kg}$ and $152 \mathrm{~cm}$, respectively. At 54 years of age, she received heart surgery for a stent placement that was followed up by aspirin treatment. At 62 years of age, her brain MRI showed an old lacunar infarct at the left thalamus and basal ganglia. Her bone density was low; she had osteoporosis, with a T-score of -3.25 and a Z-score of -0.77 . Her ophthalmologic evaluation confirmed her visual acuity, extraocular muscle movement, and optic nerves all to be normal.

\section{Treatment}

All three cases were managed with frequent feeding, intake of raw corn starch before going to bed, and L-arginine (200-300 $\left.\mathrm{mg} \cdot \mathrm{kg}^{-1} \cdot \mathrm{day}^{-1}\right)$, L-carnitine $\left(100 \mathrm{mg} \cdot \mathrm{kg}^{-1} \cdot \mathrm{day}^{-1}\right)$, coenzyme 010 (100 mg/day), and multivitamin supplementation. Case 1 has been treated frequently with intravenous glucose at her parents' hospital, for dizziness, diplopia, and droopy eyelids. Her treatment with L-arginine was started at 17 years of age. Prior to treatment, Case 1 had a lactic acid level of $6.0 \mathrm{mEq} / \mathrm{L}$. After 1 month of the L-arginine treatment combined with dietary management, she had less fatigability and was able to return to college. Her lactic acid levels were maintained at between 3.8 and 4 $\mathrm{mEq} / \mathrm{L}$

After the successful L-arginine treatment trial with Case 1, Larginine supplementation was started on cases 2 and 3 . All three cases have been treated with L-arginine for over 20 years to date. 
The most recent follow-up revealed lactic acid levels of $3.8 \mathrm{mEq} /$ $\mathrm{L}$ in Case 1 and $3.1 \mathrm{mEq} / \mathrm{L}$ in Case 3. Their alanine and arginine levels were in the range of 651 to 663 and 76 to $93 \mu \mathrm{mol} / \mathrm{L}$, respectively.

\section{Results}

\section{1) Electrocardiography}

The electrocardiograms showed possible right ventricular hypertrophy, probable acute anteroseptal infarction, ST elevation in anterior leads, and myocardial injury in Case 1 , and normal to sinus tachycardia and right axis deviation in Case 2. Case 3 showed a normal sinus rhythm.

\section{2) Pulmonary function test}

The pulmonary function test results showed a moderately restrictive pattern for cases 1 and 3 . Case 2 had normal results (Table 1).

\section{3) Audiologic evaluation}

Case 1 has sensorineural hearing impairment, whereas cases 2

Table 1. Spirometry findings showing abnormal lung parameters in cases 1 and 3

\begin{tabular}{lcccc}
\multicolumn{1}{c}{ Parameter } & Reference & Case 1 & Case 2 & Case 3 \\
\hline Vital capacity (L) & 4.5 & 1.58 & 4.3 & 1.60 \\
Inspiratory reserve volume (L) & 3.0 & 0.73 & 2.7 & 0.80 \\
\hline Tidal volume (L) & 0.5 & 0.46 & 0.5 & 0.49 \\
\hline Expiratory reserve volume (L) & 1.0 & 0.39 & 0.8 & 0.40 \\
Forced expiratory volume (\%) & $>80$ & 58 & 83 & 60 \\
Forced vital capacity (\%) & $>70$ & 57 & 70 & 68 \\
\hline
\end{tabular}

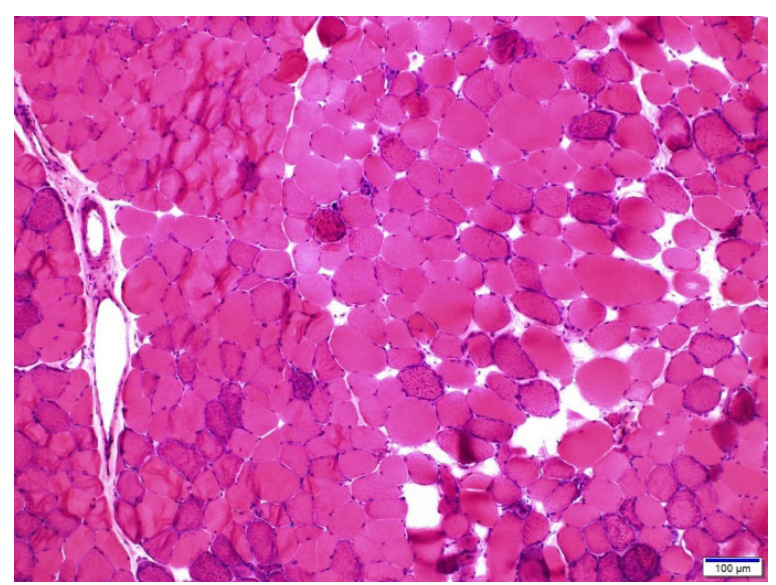

Fig. 2. Relatively uniform muscle cells with occasionally internalized nuclei. Some fibers show cytoplasmic basophilia with granularity (H\&E stain, $\times 100$ ). and 3 have normal hearing

\section{4) Bone densitometry}

The bone density remained low throughout (T-score [mean \pm standard deviation]: $-2.02 \pm 0.211 ;$ Z-score: $-1.63 \pm 0.41$ ) in all three cases. Cases 1 and 3 showed osteoporosis.

\section{5) Laboratory findings}

For all three cases, the complete blood count was normal (hemoglobin: $13.3 \mathrm{~g} / \mathrm{dL}$; platelets: $284,000 / \mu \mathrm{L}$ ), and the blood gas showed mild acidosis ( $\mathrm{pH}: 7.18$ to $7.36 ; \mathrm{pO}_{2}: 37$ to $42 \mathrm{mmHg}$ (reference, 174 to $190 \mathrm{mmHg}$ ); base excess: -11.9 to $-3.1 \mathrm{mmol} /$ $\mathrm{L}_{\mathrm{i}} \mathrm{HCO}_{3}$ : 16 to $21 \mathrm{mmol} / \mathrm{L} ; \mathrm{O}_{2}$ saturation: $99 \%$ ).

The lactic acid level (3.8 to $6.0 \mathrm{mEq} / \mathrm{L}$ [reference, 0.4 to 2.0 $\mathrm{mEq} / \mathrm{L}]$ ) was mildly elevated, the blood $\mathrm{NH}_{3}$ was normal, the lactate dehydrogenase level was 477 to $517 \mathrm{U} / \mathrm{L}$ (reference, 160 to $460 \mathrm{U} / \mathrm{L}$ ), the creatinine phosphokinase level was $243 \mathrm{U} / \mathrm{L}$ (reference, 43 to $165 \mathrm{U} / \mathrm{L}$ ), glucose level was $162 \mathrm{mg} / \mathrm{dL}$ (reference, 70 to $110 \mathrm{mg} / \mathrm{dL}$ ), and the electrolyte and uric acid levels were normal.

Biochemical and metabolic evaluations were performed on all family members. The plasma amino acid analysis revealed a substantial increase of alanine, which is associated with lactic acid elevation. The urine amino acid test showed generalized aminoaciduria, representing renal tubular dysfunction. The urine organic acid test revealed the presence of lactic acid, ethylmalonic acid, tricarboxylic acid cycle metabolites, dicarboxylic acids, tyrosyluria, and 3-methylglutaconic aciduria. The acylcarnitine profile and glycosaminoglycan levels were normal. Metabolic tests for very-long-chain fatty acids, pteridines, and dihydropteridine reductase (DHPR) were normal; that is, neopterin at 1.41

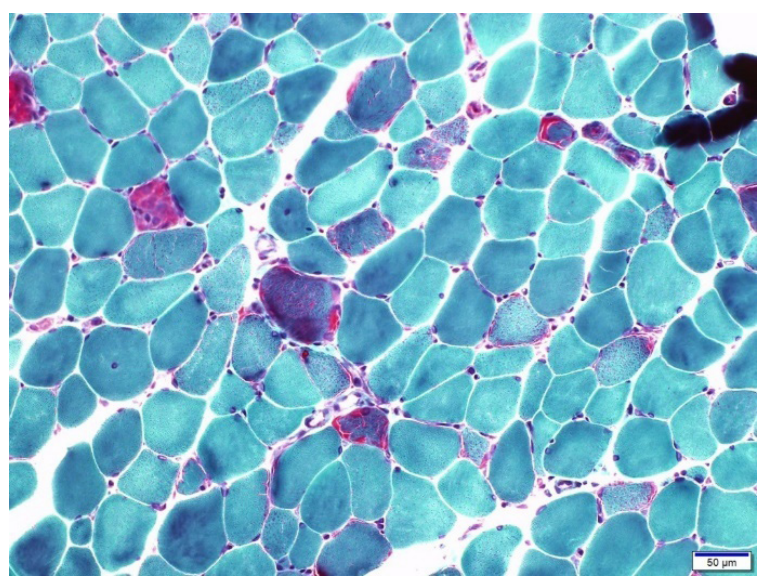

Fig. 3. Several ragged red fibers are well delineated on modified Gomori trichrome stain $(\times 200)$. 
$\mathrm{mmol} / \mathrm{mol}$-creatinine (reference, 0.23 to $0.33 \mathrm{mmol} / \mathrm{mol}$-creatinine), biopterin at $3.99 \mathrm{mmol} / \mathrm{mol}$-creatinine (reference, 0.55 to $0.87 \mathrm{mmol} / \mathrm{mol}$-creatinine), and DHPR at $3.75 \mathrm{nmol} \cdot \mathrm{min}^{-1} \cdot 5$ $\mathrm{mm} \mathrm{disc}{ }^{-1}$ (reference, $2.42-4.72 \mathrm{nmol} \cdot \mathrm{min}^{-1} \cdot 5-\mathrm{mm} \mathrm{disc}^{-1}$ ).

6) Pathologic evaluation of muscle biopsy specimens

The skeletal muscle biopsy specimens from cases 1 and 2 showed very similar morphologic changes. The H\&tE stains revealed relatively uniformly sized muscle cells with occasional internalized nuclei. Some fibers showed cytoplasmic basophilia with a granular appearance (Fig. 2). Upon modified GT staining, several RRFs were observed (Fig. 3). Ultrastructurally, subsarcolemmal collections of abnormally sized and shaped mitochondria with inclusions were evident (Fig. 4). Electron microscopy of the muscle showed mitochondria with enlarged, elongated,

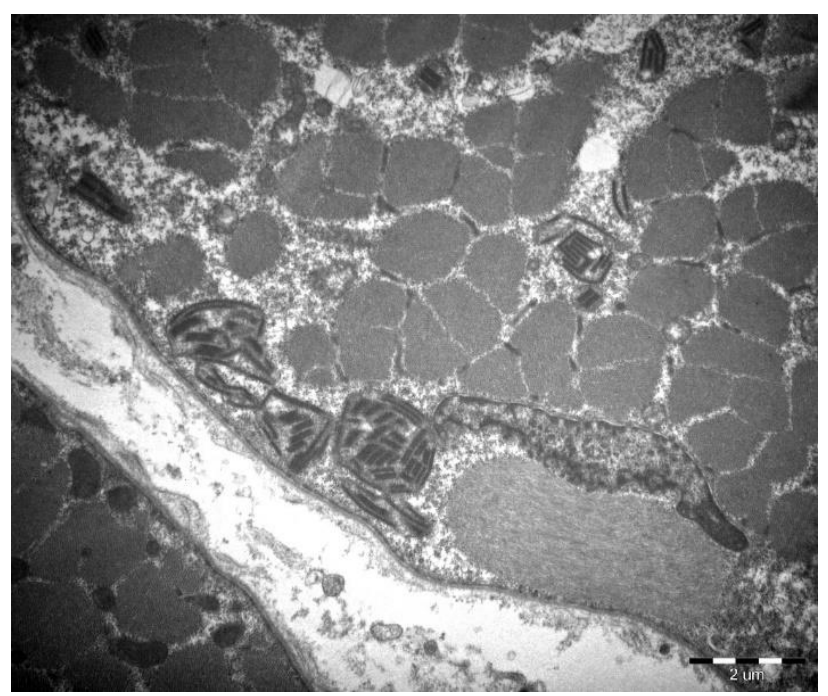

Fig. 4. Subsarcolemmal collection of abnormal mitochondria with inclusions (transmission electron microscope, $\times 12,000$ ).

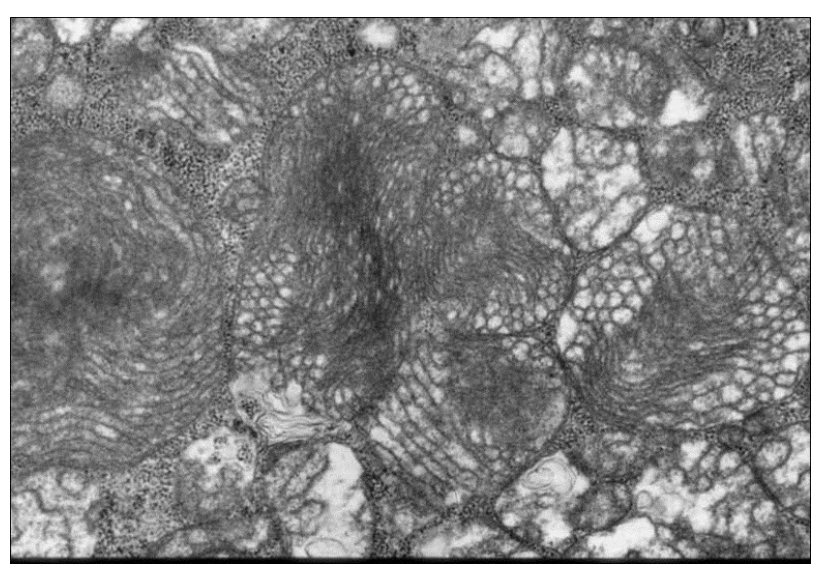

Fig. 5. Thread bundle shape of mitochondria. concentric, and thread-like bundle shapes (Fig. 5). Some mitochondria showed thickened onion-skin-like cristae and contained parking-lot-shaped crystalline and paracrystalline inclusions (Fig. 6).

Case 3 chose not to undergo a muscle biopsy.

\section{7) Brain imaging}

Case 1 had a head computed tomography scan in 1997 (at 17 years of age) because of recurrent headaches, but the findings were normal. The brain MRI findings for Case 2 at 36 years of age were normal except for mild platybasia. There were no areas of restricted diffusion or evidence of an acute infarct. There was no evidence of a brain parenchymal lesion or abnormal signal intensity. The brain MRI of Case 3 (Fig. 7), taken when she was 62 years old, showed an old lacunar infarct at the left thalamus and basal ganglia.

\section{8) Molecular tests}

Molecular studies were performed on skin fibroblast specimens. All three cases had the same mtDNA mutation. The mother (Case 3) had the lowest mutation load (Table 2).

\section{Discussion}

Because MELAS syndrome affects multiple organs and has a broad range of possible clinical manifestations, it can be difficult to diagnose. Patients with this disorder can have poor appetite, motion sickness, migraines, ptosis, fatigability, and neurologic deficits due to stroke-like events. Acute presentations of MELAS can be mistaken for myasthenia gravis, vascular strokes, epilepsy,

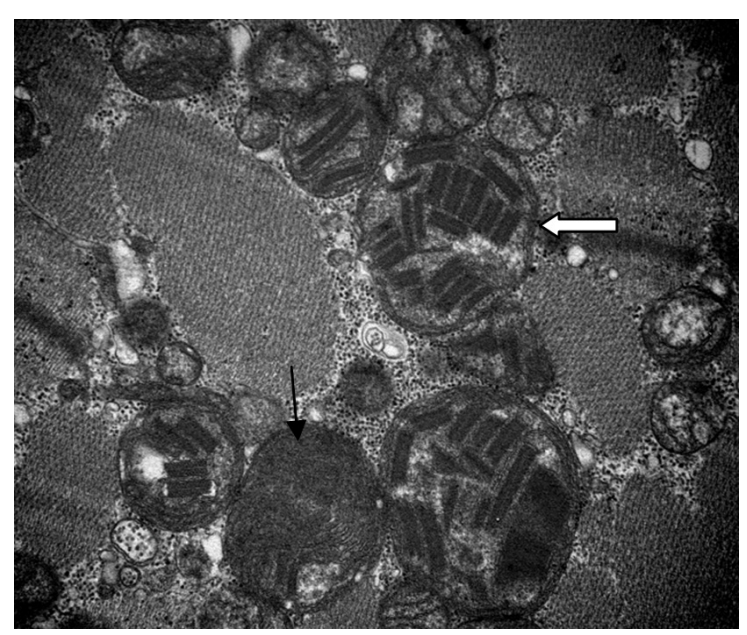

Fig. 6. Onion skin cristae (black arrow) and several enlarged mitochondria containing parking lot shaped inclusions (white arrow) (transmission electron microscope, $\times 30,000$ ). 

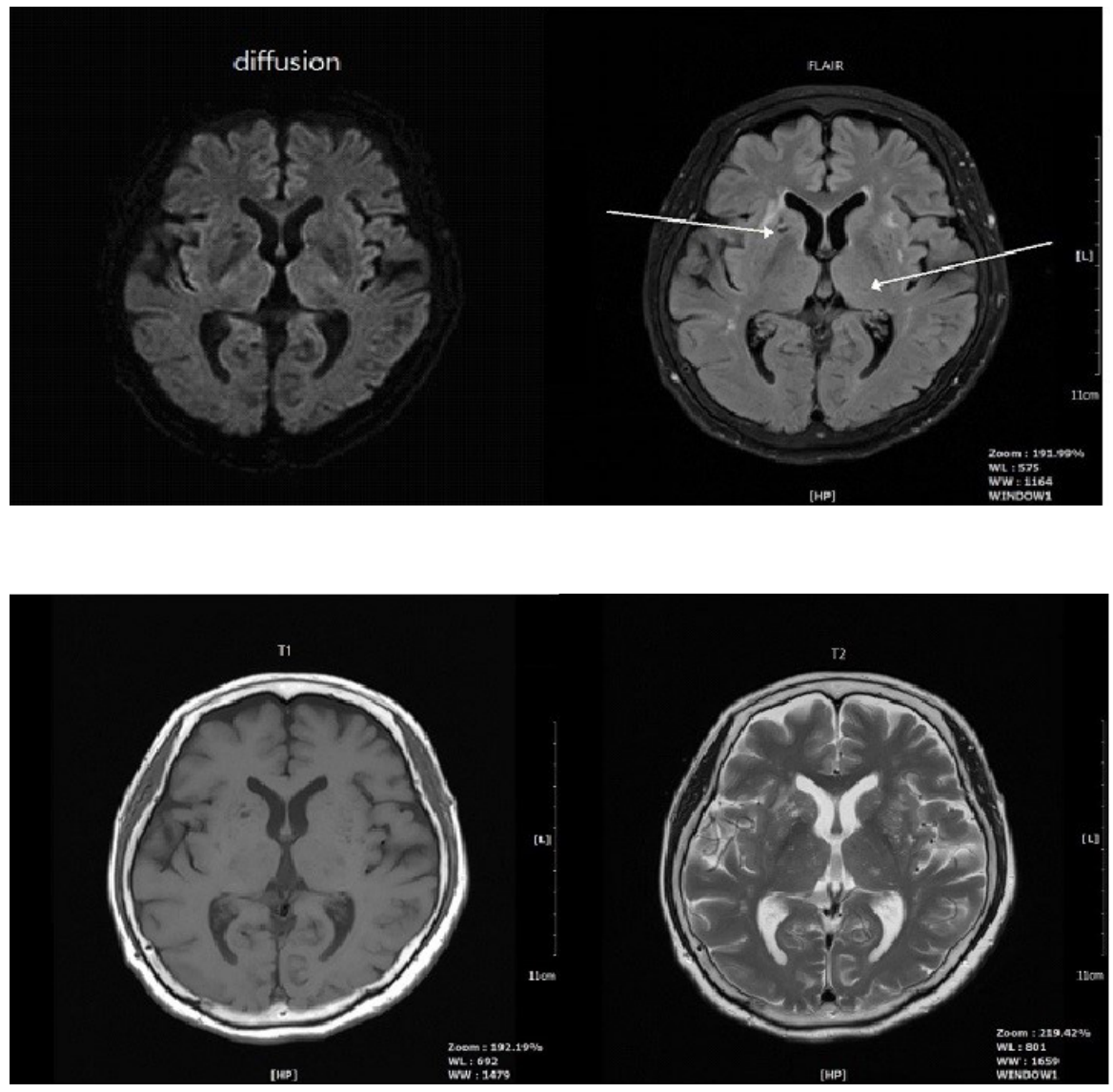

Fig. 7. Case 3 at age of 62 years. Her brain magnetic resonance imaging showed old lacunar infarct at left thalamus and basal ganglia (arrows).

Table 2. Identical mitochondrial DNA (mtDNA) mutation and different mutation loads observed in the family

\begin{tabular}{ccc}
\hline Case & mtDNA mutation & Mutant Load of tRNA ${ }^{\text {Leu }}(\%)$ \\
\hline 1 & $3243 A>G$ & 20 \\
2 & $3243 A>G$ & 13 \\
3 & $3243 A>G$ & 10 \\
\hline
\end{tabular}

encephalopathy, infection, psychosis, and other neurodegenerative disorders [7]. The first presentations of afflicted patients are often nonspecific and seldom exhibit the numerous complications associated with MELAS, and the syndrome can take decades to fully develop [8]. Furthermore, there is significant clinical heterogeneity in the patients with the same mutation or those within the same family.

Our Case 1 patient was initially misdiagnosed with myasthenia gravis and had sought medical attention because of the severe side effects of a cholinesterase inhibitor given to treat that condition. Furthermore, because of the misdiagnosis, a mass in her thymus was thought to be a thymoma associated with myasthenia gravis. A Tensilon test and an acetylcholine receptor antibody test would have helped to provide an earlier diagnosis of MELAS and prevented the unnecessary suffering of the patient.

The point mutation at $m+D N A 3243 A>G$ is highly specific to the disease, being prevalent in 84-100\% of cases [9], and a very important diagnostic clue. Although molecular techniques play a critical role in the diagnosis of respiratory chain diseases, we believe the evidence of RRFs is one of the cornerstones in the diagnosis of MELAS. If the modified GT stain shows the characteristic RRFs, it can help to rule out ophthalmoplegia, KearnsSayre syndrome, and myoclonic epilepsy associated with ragged red fiber syndrome. However, RRFs can also occur in other neuromuscular disorders, such as muscular dystrophy, polymyositis, dermatomyositis, and some glycogenoses. Therefore, the combination of both clinical and histochemical features is important for a definitive diagnosis. Regions of ischemic damage traversing multiple cerebrovascular territories suggest that an underlying cause is linked to the energy production disruption or microvasculature, rather than the more common pattern of large-vessel involvement associated with cardiovascular causes. Whereas 
the tissue vulnerability threshold may not differ substantially among individuals, the mutation load and tissue distribution do vary and may account for the clinical diversity seen in individuals with MELAS. Correlations between the frequency of more common clinical features and the level of mutant mtDNA in the muscle, but not in leukocytes, have been observed $[10,11]$.

Patients with MELAS usually have a positive family history. Therefore, when present with other indicators of mitochondrial dysfunction, such as hearing loss, myopathy, or diabetes that runs in the family, MELAS must be considered regardless of age, and underlying mitochondrial derangements should be investigated through a molecular workup [3].

Several studies have reported rapidly progressive deterioration of the sensorineural function, cardiac left ventricular hypertrophy, and overall severity of symptoms as well as electroencephalogram abnormalities in all individuals affected with MELAS $[12,13]$. However, the onset of stroke-like episodes in MELAS varies greatly, where approximately $70 \%$ of individuals present with these before age 20 years, and over $90 \%$ present them before age 40 years $[3,5]$. Case 3 did not come to medical attention until MELAS was diagnosed in Case 1. Therefore, the exact onset of the first stroke-like episode in Case 3 is not clear, but her stroke was first discovered at the age of 62 years.

The reason for the late onset of neurologic symptoms in certain patients with MELAS may be the mtDNA variant load [5]. The relatively low rate of heteroplasmy in the mother (10\%) might have contributed to her late symptom onset and overall mild clinical course. The heteroplasmy rates of this patient's muscle and brain tissue, which may be more predictive of the disease course and severity, are unknown.

Ophthalmologic symptoms appear earlier in MELAS. For Case 1 , it was at age 9 years, whereas it was at 11 years for Case 2 .

Effective management must consider the metabolic and ischemic aspects of MELAS. Mitochondrial dysfunction, particularly impaired neuronal oxidative energy production, results in a high degree of non-oxidative glycolysis, as indicated by the elevated lactic acid levels in the serum and cerebrospinal fluid of patients with MELAS $[2,14]$. Cerebral hypoperfusion results in hypoxemic damage to the neurons, which are particularly susceptible to ischemic insults. The pathologic mechanism behind the strokelike episodes and complications is small vessel angiopathy due to compensatory mitochondrial proliferation in the endothelium and vascular smooth muscle and reduced production of the vasodilator nitric oxide.

Genetic counseling is highly recommended, as all children of an affected female will inherit the mutation. Genetic coun- selors are able to assist patients and their families with family planning, preimplantation genetic diagnosis, and a pronuclear transfer option if it becomes available in Korea. However, the unpredictable levels of the mutation load and the varying distributions of the mutated mitochondria in organs and tissues lead to a wide range of clinical manifestations, making it very difficult to determine the severity of the disease [11]. Case 2 participated in a family planning session and has a healthy son as his spouse was free from the mitochondrial disorders.

Most importantly, these cases of MELAS show the importance of a prompt diagnosis, as early intervention can facilitate optimal management of the condition and help improve outcomes. In particular, recent evidence indicates that supplementation with the nitric oxide precursors L-arginine and L-citrulline may offer greater protection if started earlier in life $[15,16]$. It is hypothesized that L-citrulline and L-arginine supplementation increase the nitric oxide availability, improve endothelial function and cerebral blood flow, and reduce the incidence and severity of stroke-like episodes. Although the mother (Case 3) did not come to medical attention with neurologic symptoms of MELAS until she was 49 years old, she has nonetheless done well with long-term metabolic supplementation.

In summary, we have described three familial cases of genetically confirmed MELAS. The only individual with stroke-like episodes was the mother, at the age of 62 years. One of her brothers had died from a sudden cardiac arrest, and her mother suffered from dementia, myocardial infarction, and diabetes. Other siblings of Case 3 are so far mostly non-symptomatic. She had heart stent placement surgery at 54 years of age and a lacunar stroke at 62 years of age. Compared with previously reported cases with MELAS, our patients, who have been on dietary supplementation for 21 years, have had relatively mild symptoms and a slowly progressing clinical course.

In conclusion, when myasthenia gravis is suspected, it is important to rule out MELAS or other mitochondrial diseases as early as possible, because a prompt diagnosis and early prevention may improve the disease outcomes. Frequent feeding, a nightly raw corn starch diet, and L-carnitine, coenzyme 010, Larginine, and multivitamin supplementation appear to be an essential part of good long-term management for MELAS.

Prompt diagnosis upon the initial presentation of MELAS is crucial, as early intervention may improve outcomes. Recent evidence and our 21-year-long experience indicate that supplementation with the nitric oxide precursors L-arginine and Lcitrulline offers greater protection from stroke-like episodes if started earlier in life. When facing what appears to be myasthe- 
nia gravis, more steps must be taken to distinguish it from other possible causative mitochondrial or autoimmune disorders.

\section{Acknowledgements}

The authors would like to thank the patients, and Progen Inc. for performing molecular studies.

\section{References}

1. Mauro SD, Hirano M. MELAS synonyms: mitochondrial encephalomyopathy, lactic acidosis, and stroke-like episodes; myopathy, mitochondrial-encephalopathy-lactic acidosis-stroke. In: Adam MP, Ardinger $\mathrm{HH}$, Pagon RA, Wallace SE, eds. GeneReviews ${ }^{\circledR}$ [Internet]. Seattle (WA): University of Washington, 2001 Feb 27 [updated 2018 Nov 29; cited 2018 Dec 20]. [https://www.ncbi.nlm.nih.gov/books/ NBK1233/]

2. Hirano $M$, Ricci $E$, Koenigsberger MR, Defendini R, Pavlakis SG, DeVivo DC, et al. MELAS: an original case and clinical criteria for diagnosis. Neuromuscul Disord 1992;2:125-35.

3. Jeppesen TD, Schwartz M, Hansen K, Danielsen ER, Wibrand F, Vissing J. Late onset of stroke-like episode associated with a 3256C-->T point mutation of mitochondrial DNA. J Neurol Sci 2003;214:17-20.

4. Kisanuki $Y Y$, Gruis KL, Smith $T L$, Brown DL. Late-onset mitochondrial myopathy, encephalopathy, lactic acidosis, and strokelike episodes with bitemporal lesions. Arch Neurol 2006;63:1200-1.

5. Parikh S, Goldstein A, Koenig MK, Scaglia F, Enns GM, Saneto R, et al. Diagnosis and management of mitochondrial disease: a consensus statement from the Mitochondrial Medicine Society. Genet Med 2015;17:689-701.

6. Wiwatwongwana A, Lyons CJ. Eye movement control and its disorders. In: Dulac O, Lassonde M, Sarnat HB, eds. Pediatric neurology, part III: handbook of clinical neurology. Amsterdam: Elsevier,
2013;1505-13.

7. Leff AP, McNabb AW, Hanna MG, Clarke CR, Larner AJ. Complex partial status epilepticus in late-onset MELAS. Epilepsia 1998;39:438-41.

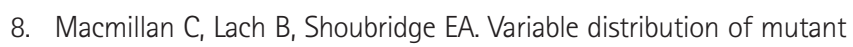
mitochondrial DNAs (tRNA(Leu[3243])) in tissues of symptomatic relatives with MELAS: the role of mitotic segregation. Neurology 1993;43:1586-90.

9. Bataillard $M$, Chatzoglou $E$, Rumbach $L$, Sternberg D, Tournade A, Laforêt $P$, et al. Atypical MELAS syndrome associated with a new mitochondrial tRNA glutamine point mutation. Neurology 2001;56:405-7.

10. Jeppesen TD, Schwartz M, Frederiksen AL, Wibrand F, Olsen DB, Vissing J. Muscle phenotype and mutation load in 51 persons with the 3243A>G mitochondrial DNA mutation. Arch Neurol 2006;63:1701-6.

11. Chinnery PF, Howell N, Lightowlers RN, Turnbull DM. Molecular pathology of MELAS and MERRF. The relationship between mutation load and clinical phenotypes. Brain 1997;120:1713-21.

12. Majamaa-Voltti KA, Winqvist $S$, Remes AM, Tolonen $U$, Pyhtinen J, Uimonen $S$, et al. A 3-year clinical follow-up of adult patients with 3243A>G in mitochondrial DNA. Neurology 2006;66:1470-5.

13. Yatsuga S, Povalko N, Nishioka J, Katayama K, Kakimoto N, Matsuishi T, et al. MELAS: a nationwide prospective cohort study of 96 patients in Japan. Biochim Biophys Acta 2012;1820:619-24.

14. Wilichowski E, Pouwels PJ, Frahm J, Hanefeld F. Quantitative proton magnetic resonance spectroscopy of cerebral metabolic disturbances in patients with MELAS. Neuropediatrics 1999;30:256-63.

15. El-Hattab AW, Emrick LT, Hsu JW, Chanprasert S, Almannai M, Craigen WJ, et al. Impaired nitric oxide production in children with MELAS syndrome and the effect of arginine and citrulline supplementation. Mol Genet Metab 2016;117:407-12.

16. Koga Y, Akita Y, Nishioka J, Yatsuga S, Povalko N, Tanabe Y, et al. LArginine improves the symptoms of strokelike episodes in MELAS. Neurology 2005;64:710-2. 